\title{
Redressing the balance in autism research
}

\author{
Patricia Howlin
}

The Department of Health in the UK has recently announced its "Adult Autism Strategy", with funding of $£ 0.5$ million, to explore the numbers of adults with autism spectrum disorders in the UK and their needs for services. The budget, though small, is to be welcomed; but why, some may ask, is yet more money being earmarked for autism, probably the most widely researched and most generously funded of all developmental disorders?

In the US, the NIH funds eight dedicated "Centers of Excellence in Autism Research" to bring together researchers and the resources they need. The US-based charity Autism Speaks provides millions of dollars in funding each year for autism research. In the UK, the Medical Research Council provides around £1.5-2 million per annum for the same purpose. Thanks to such funding we now know much more about the prevalence of autism spectrum disorders in childhood (approximately 1\%; Baird G et al. [2006] Lancet 368: 210-215). Diagnosis can be made reliably by around 2 years of age, and there is increasing evidence to support the effectiveness of early intervention programs.

However, almost all funded research focuses on children with the disorder, with family-based and psychoeducational programs concentrating particularly on children under the age of 6 years. Of over 100 autism trials currently listed by the US NIH (www.clinicaltrials.gov/ct2), only threeall drug trials - focus specifically on adults.

Unfortunately, autism does not come to an abrupt end at the age of 6 years - it is a lifelong disorder and there are far more adults than children living with an autism spectrum disorder. Nevertheless, not only does the funding of research into interventions for people with autism spectrum disorders diminish dramatically from later childhood onwards, so too do service provision and clinical expertise. In the UK, generic 'learning disability' services are available for adults with autism spectrum disorders whose IQ is below 70 , an arbitrary and ludicrous barrier for determining eligibility;
It is now

crucial that

the research

effort currently

concentrated

on children

is expanded

to meet the

needs of adults

with autism

spectrum

disorders...

P Howlin is Professor of Clinical Child

Psychology at the

Institute of Psychiatry, King's College London, London, UK.

Competing interests

The author declared no

competing interests.

www.nature.com/clinicalpractice doi:10.1038/ncpneuro0860 for the majority, who have an IQ above this level, no statutory services exist. Any help that individuals with autism spectrum disorders and their families may have previously received from Child and Adolescent Mental Health Services and educational services is also withdrawn from the age of 18 years. Many people with an autism spectrum disorder and their parents describe feeling as if they have suddenly fallen into a "bottomless pit". Specialist guidance to help people with autism spectrum disorders through college is sparse, dedicated employment services are rare, and support for independent living is nonexistent in much of the UK. The situation in other countries tends to be similar.

The result is that many adults with an autism spectrum disorder remain almost entirely dependent on their steadily aging parents for support; they are socially isolated, economically unproductive, and financially disadvantaged. It is hardly surprising that rates of mental health problems, particularly those related to anxiety and depression, are estimated to be as high as $30 \%$ in this group. For society, too, the economic implications are huge, with estimates of the costs for adults with autism spectrum disorders in the UK being around £25 billion per annum (Knapp M et al. [2007] The Economic Consequences of Autism. London: Mental Health Foundation). There is evidence that, with appropriate support, much can be donededicated employment schemes, for example, can have a great impact on the numbers and level of jobs obtained (Howlin P et al. [2005] Autism 9: 533-549). However, funding for such schemes tends to be short-term and sporadic.

It is now crucial that the research effort currently concentrated on children is expanded to meet the needs of adults with autism spectrum disorders and their families. Let us hope that the latest initiative by the UK government will pave the way to high-quality, adequately funded research, and to improved service provision for this largely neglected section of the population. 